\title{
Calibration of the LOFAR antennas
}

\author{
J.R. Hörandel ${ }^{* 1,2}$, S. Buitink ${ }^{3}$, A. Corstanje ${ }^{1}$, J.E. Enriquez ${ }^{1}$, H. Falcke ${ }^{1,2,4}$, \\ T. Karskens ${ }^{1}$, M. Krause ${ }^{1,5}$, A. Nelles ${ }^{1,6}$, J.P. Rachen ${ }^{1}$, L. Rossetto ${ }^{1}$, P. Schellart ${ }^{1}$, \\ O. Scholten ${ }^{7,8}$, S. ter Veen ${ }^{1,4}$, S. Thoudam ${ }^{1}$, T.N.G. Trinh ${ }^{7}$
}

1 Department of Astrophysics/IMAPP, Radboud University Nijmegen, P.O. Box 9010, 6500 GL Nijmegen, The Netherlands

2 NIKHEF, Science Park Amsterdam, 1098 XG Amsterdam, The Netherlands

3 Astrophysical Institute, Vrije Universiteit Brussel, Pleinlaan 2, 1050 Brussels, Belgium

4 Netherlands Institute of Radio Astronomy (ASTRON), Postbus 2, 7990 AA Dwingeloo, The

Netherlands

5 Now at: DESY, Platanenallee 6, 15738 Zeuthen, Germany

6 Now at: Department of Physics and Astronomy, University of California Irvine, Irvine, CA 92697-4575, USA

7 KVI-CART, University Groningen, P.O. Box 72, 9700 AB Groningen, The Netherlands

8 Interuniversity Institute for High-Energy, Vrije Universiteit Brussel, Pleinlaan 2, 1050

Brussels, Belgium

E-mail: j.horandeleastro.ru.nl

\begin{abstract}
Extensive air showers create short nanosecond-scale pulses in the radio frequencies. These pulses have been measured successfully in the past years at the Low-Frequency Array (LOFAR). Due to the short duration and emission of the signal in the atmosphere, methods based on flux calibration of known sources as used in radio astronomical observations cannot be applied to establish an absolute calibration. To overcome this, we present three approaches that were used to check and improve the antenna model of LOFAR, and to provide an absolute calibration for air shower measurements. Two include the calibration with made-made sources, while one is using the Galactic radio emission. A source has been used that allows the cross-calibration of LOPES, Tunka-Rex and LOFAR. In future work these results can be used as an absolute scale for measurements of astronomical transients with LOFAR.
\end{abstract}

The 34th International Cosmic Ray Conference,

30 July- 6 August, 2015

The Hague, The Netherlands

\footnotetext{
* Speaker.
} 


\section{Introduction}

Radio detection of cosmic rays has been established as a detection technique in the past years. The emission mechanism of the radiation is understood and full Monte Carlo simulations are able to predict the emission in great detail. One of the remaining challenges is the absolute calibration of the antenna systems. Since there is, not yet, such a thing as a "single muon peak" in radio detection, calibration methods have to be defined that are easy to use and allow for a comparison of the measured field-strengths, and therefore, the air shower energies measured with different experiments.

At LOFAR, the efforts of several groups have been combined. Our colleagues form the Pierre Auger Observatory have provided their octocopter to allow us to study the directional dependency of the antennas [1]. The radio quiet background at LOFAR, provided us with excellent conditions to use the diffuse Galactic emission as calibration source. Also, the same reference source as used at LOPES and Tunka-Rex, which is suspended from a crane, has been brought to LOFAR to cross-calibrate the absolute scale of the three experiments [2, 3].

In these proceedings, we will summarize the measurements and calculations as they are presented in detail in [4].

\section{Analysis methods}

Every analysis of the radio emission of air shower, requires several ingredients to correct for the influence on the hardware used. The sensitivity of the antenna used, is not uniform in the angles $\theta$ and $\phi$. Depending on the polarization of the signal, the antenna will only detect signals from certain directions. These complex patterns are usually simulated in full electromagneticsimulations and referred to as antenna model. The antenna model has the units of an effective height, as the antenna converts the electric field emitted by an air shower to a voltage.

After the antenna, the signals are amplified and filtered. One needs to correctly account for these components to obtain an absolute scale for the measurements. One should thereby note that all quantities are functions of frequency and contain both an amplification value (gain), as well as a group delay (phase) that is introduced by every component. This makes the sensitivity of radio antenna systems a function of several parameters that have to considered during calibration.

\section{Directivity of the antenna sensitivity}

Figure 1 shows the set-up used for the octocopter calibration. A network analyzer has been connected to the footpoint of a LOFAR low-band antenna (LBA), as well as to a transmission antenna. In this way, the frequency response of the antenna has been be tested as function of angle around the antenna. The results are shown in Figure 2.

The antenna model is shown to reasonably follow the measurements, both as function of frequency and zenith angle. There are, however, some discrepancies. These discrepancies are likely due to an incomplete modeling of the antenna, which is drawback of the current model that only includes simplified models of a number of components. Furthermore, rain and a varying ground conditions are known to influence the antenna response, which is also not accounted for. 


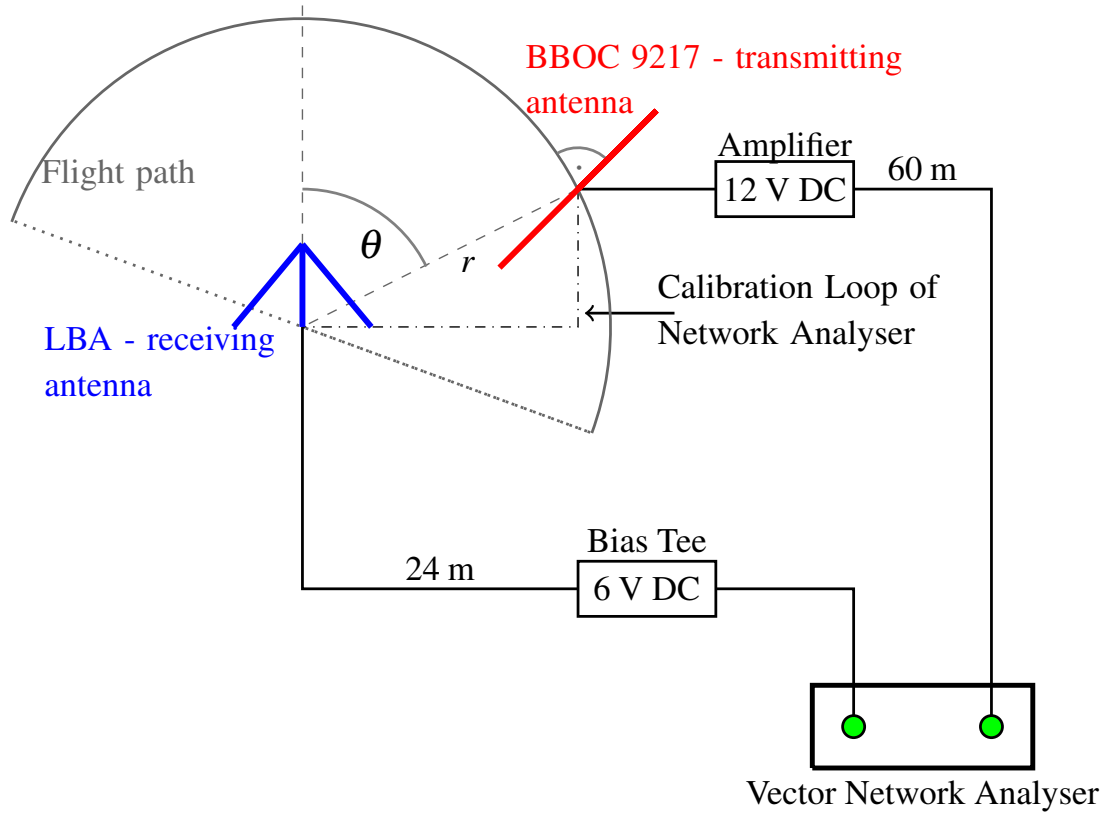

Figure 1: Schematic drawing of the experimental set-up with the flying source calibration in a closed loop. The grey half circle denotes the path of the transmitting antenna. The angle $\theta$ highlights the zenith angle of the transmitting antenna with respect to the LBA. The black dashed-dotted line indicates the cable connection between the LBA and the amplifier used for the calibration of the network analyser.

Due to the complexity of the LOFAR system that allows for switching of observation modes and antenna types through software, it is extremely difficult to measure all filters and amplification stages separately. Therefore, it is thought to be easiest to have an end-to-end calibration of the system. For such an end-to-end calibration, the antenna model needs to only be a fair approximation of the real response. The measurements with the octocopter indicates that this is the case. The deviations will be handled in the absolute calibration.

\section{Absolute calibration of the LOFAR system}

Figure 3 shows the experimental set-up of the end-to-end calibration using a reference source on a crane. To obtain the calibration the same read-out of the LOFAR antennas is used that is also used during air shower measurements. By using a calibrated source antenna, the measured voltages in the system can be predicted and compared to the measurements. The ratio of those two provides the absolute calibration.

Figure 4 shows the Galactic emission and how it is used to calibrated the LOFAR antennas. Since the LOFAR antennas are sky noise dominated, a variation caused by the movement of the Galaxy through the antenna sensitivity is visible in the noise floor. The radio emission is calculated from the combination of emission model and antenna response and compared to the measured emission per antenna. Since the Galaxy is moving through the antenna directivity, several angles are probed. The prediction and the measurement show a convincing agreement in shape. If this 

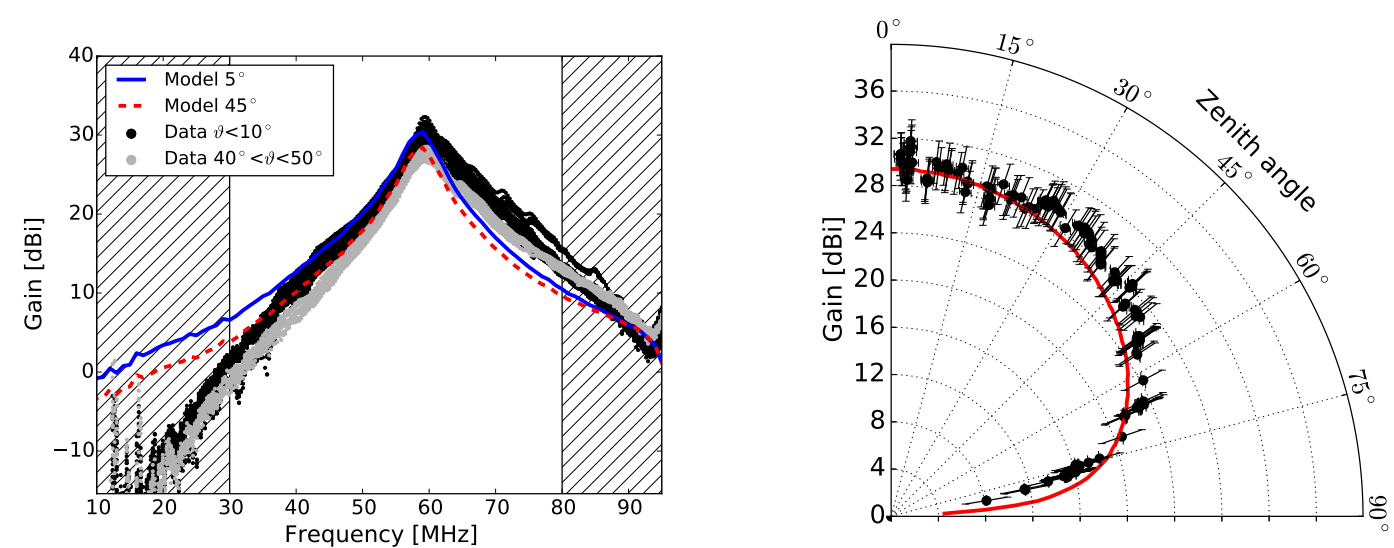

Figure 2: Left: Several gain spectra as measured with an LBA in combination with the spectrum analyzer attached to the octocopter drone. The spectra are shown for two zenith angle bins $\left(\theta<10^{\circ}\right.$ in black and $40^{\circ}<\theta<50^{\circ}$ in gray) for the complete LBA band of $10-95 \mathrm{MHz}$. The lines indicate the gain values of the antenna model for the corresponding zenith angles of $5^{\circ}$ (blue solid line) and $45^{\circ}$ (red dashed line). Additional filtering outside of the band of $30-80 \mathrm{MHz}$ is not taken into account in the current model and indicated by the shaded regions. Right: Gain factor of the antenna as a function of the zenith angle of the incoming signal at $60 \mathrm{MHz}$. The black points show the measured data averaged over several frequency scans. The red line indicates the predicted gain of the antenna model.

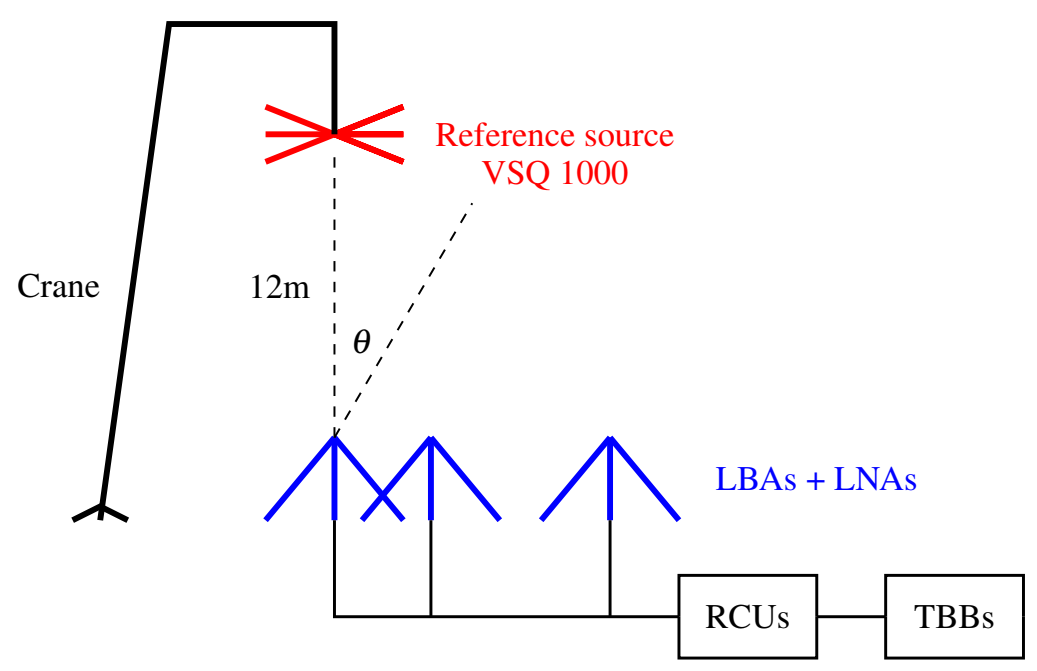

Figure 3: Schematic illustration of the experimental set-up using the stationary source calibration. The source is suspended from a crane at about $12 \mathrm{~m}$ above the chosen antenna. The signal is received with the LOFAR LBA antennas and filtered and digitized at the receiver units (RCUs). The data of all antennas of a LOFAR station are read-out via the LOFAR system using the transient buffer boards (TBB) and it is done for cosmic ray measurements. 


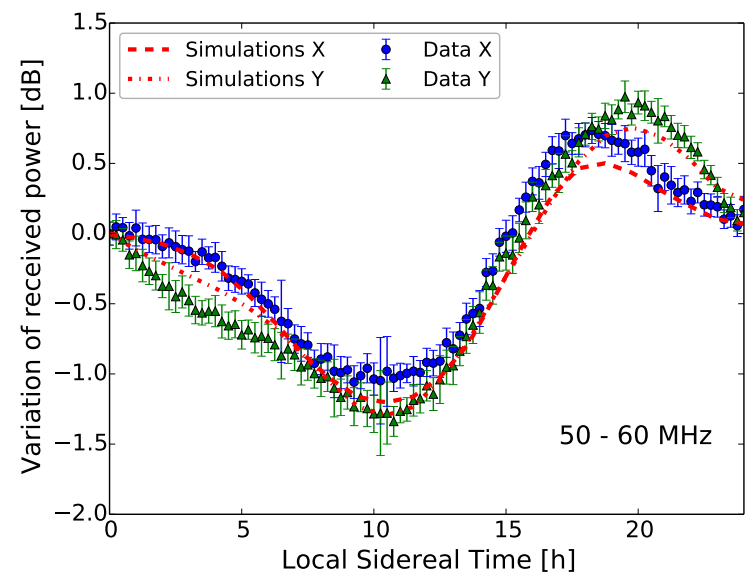

Figure 4: Variation in the measured power per antenna as function of local sidereal time in the band of $50-60 \mathrm{MHz}$. Overlaid are the predicted values from Galactic emission model and antenna response, as well as the actual measurements. The emission of the Galaxy is modelled with LFmap [5] and includes a correction for the intrinsic noise of the system.

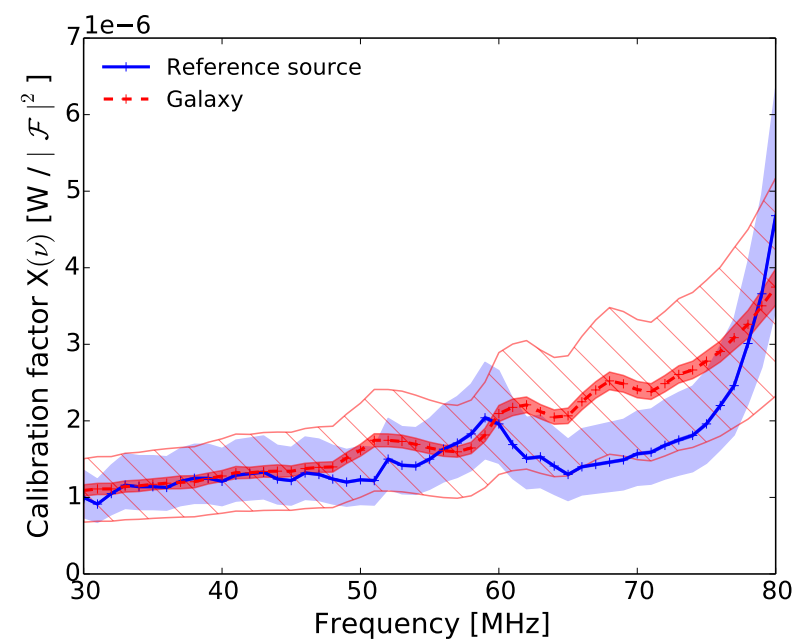

Figure 5: Calibration factors $X$ as a function of frequency across the LOFAR band for Galactic and reference source calibration. Both calibration curves contain statistical uncertainties of the method in the dark region, with systematic uncertainties illustrated by the lighter region (dashed for Galactic, filled for terrestrial).

comparison is made for all frequencies, a frequency dependent calibration curve can be obtained, similar to the one from the reference source.

Figure 5 shows the absolute calibration curves obtained with the two different methods. These curves can now be applied to air shower data, after they have been corrected for the antenna response. Both curves are in good agreement. For a detailed discussion of the observed differences, please refer to [4]. The systematic uncertainties are limited by the absolute calibration of the reference source and the system noise correction, respectively. They are about $35 \%$ in amplitude for both methods. 


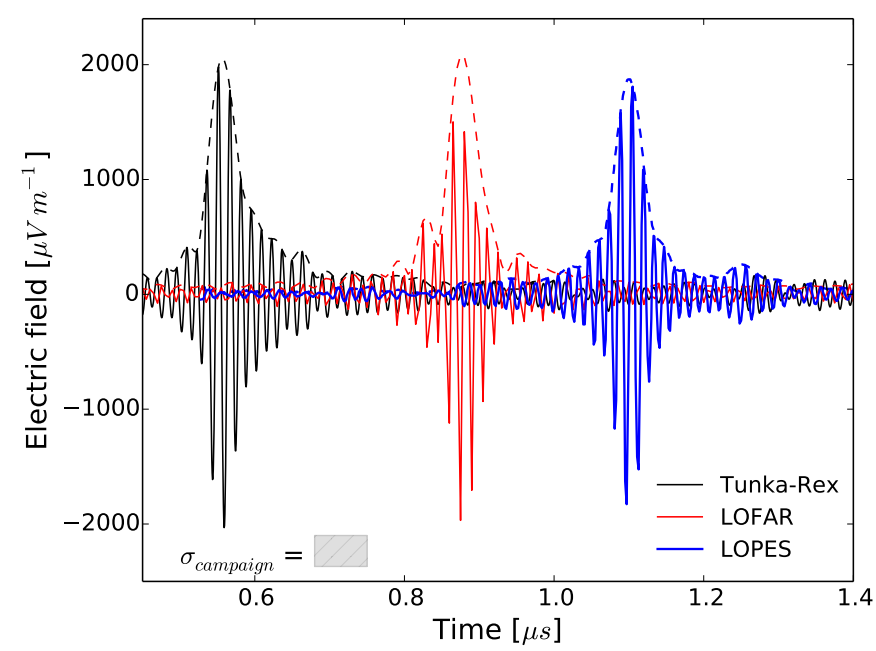

Figure 6: Electric field as function of frequency for the three calibration measurements at (from left to right) Tunka-Rex, LOFAR, and LOPES, with amplitudes scaled to match a source at 10 meter distance, up-sampled and filtered to a common band of $43-74 \mathrm{MHz}$. The dashed lines represent the calculated Hilbert envelopes for the three pulses. The size of the systematic campaign specific uncertainty on the maximum amplitude is indicated with the grey bar. These uncertainties are expected to be of the same order of magnitude for Tunka-Rex and LOPES.

\section{Comparison to other experiments and air shower simulations}

The same reference source was used at LOPES and Tunka-Rex. Figure 6 illustrates the calibration signal as measured in the three different experiments. Starting from this measurement, the method-specific absolute scale uncertainties of 33\% can be ignored, when comparing the energy scale of LOFAR, LOPES and Tunka-Rex. Thus, air shower simulation codes can now also be tested more precisely with respect to the absolute scale.

In a very simple analysis, the testing of the absolute scale has been done at LOFAR. 50 air showers were used to obtain a scaling factor between uncalibrated LOFAR data and CoREAS simulations [6]. This scaling factor is non-frequency dependent and therefore only a first indication. The two calibration methods as introduced above can also be used to obtain a scaling factor of the same type. The three scaling factors are shown in Figure 7.

In future work, we will explore the frequency dependence and thereby reduce the bands of systematic uncertainties that now limit the comparison of the three methods and the testing of the absolute scale.

\section{Conclusions}

We have established an absolute scale for the radio measurement of air showers at LOFAR with different methods. The methods are consistent with each other and with predictions from air shower simulations.

While the calibration on made-made sources allows to reduce systematic uncertainties between measurements of different experiments, these measurements are time-consuming and ask 


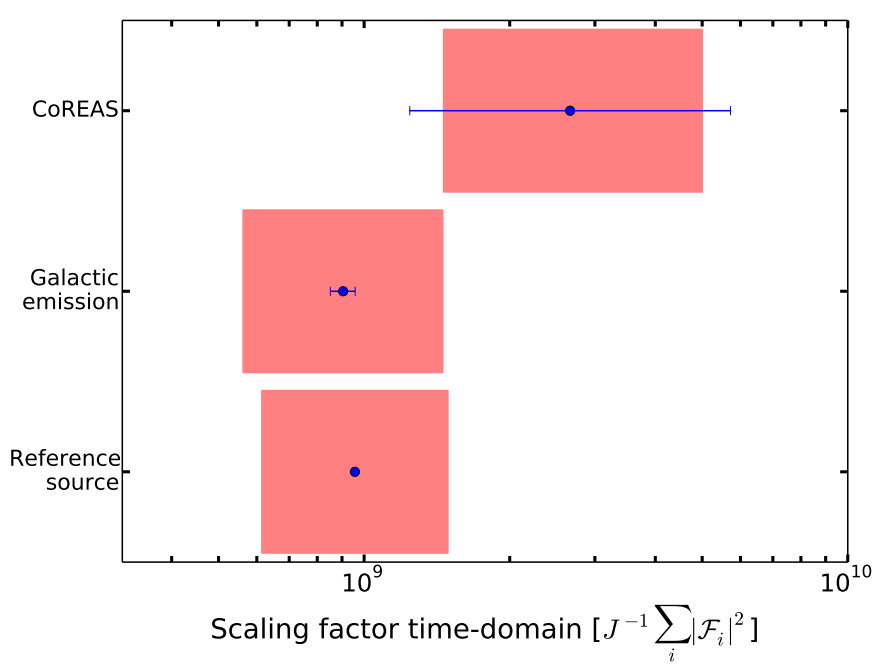

Figure 7: Scaling factor $S$ as obtained in the time-domain for the air shower simulations (CoREAS) and the two calibration methods (Galaxy and Reference source). Depicted values and uncertainties are the average and its standard deviation obtained from different events. The colored bands correspond to systematic uncertainties on the used method as discussed in the corresponding sections.

for accessibility of the antenna array. In cases of large and/or remote arrays, the calibration on the emission of the Galaxy seems a more practicable approach and yields compatible results.

The new absolute scale will be used to test models of air shower emission in detail at LOFAR. Furthermore, this calibration will be used to calculate an absolute flux of transient astronomical sources, like pulsars, which are considered challenging to measure in absolute fluxes. This step will increase the synergy between astronomy and astroparticlephysics at LOFAR.

\section{Acknowledgement}

We thank our colleagues from the Pierre Auger Collaboration, LOPES Collaboration and Tunka Collaboration for sharing their calibration methods and instruments with us.

The LOFAR cosmic ray key science project acknowledges funding from an Advanced Grant of the European Research Council (FP/2007-2013) / ERC Grant Agreement n. 227610. The project has also received funding from the European Research Council (ERC) under the European Union's Horizon 2020 research and innovation programme (grant agreement No 640130). We furthermore acknowledge financial support from FOM, (FOM-project 12PR304) and NWO (VENI grant 639041-130). AN is supported by the DFG (research fellowship NE 2031/1-1).

LOFAR, the Low Frequency Array designed and constructed by ASTRON, has facilities in several countries, that are owned by various parties (each with their own funding sources), and that are collectively operated by the International LOFAR Telescope foundation under a joint scientific policy.

\section{References}

[1] Pierre Auger Collaboration, P. Abreu et al., Antennas for the detection of radio emission pulses from 
cosmic-ray induced air showers at the Pierre Auger Observatory, Journal of Instrumentation 7 (Oct., 2012) 11P, [arXiv:1209.3840].

[2] LOPES Collaboration, S. Nehls et al., Amplitude calibration of a digital radio antenna array for measuring cosmic ray air showers, Nucl. Inst. Meth. A 589 (2008) 350-361.

[3] Tunka Collaboration, R. Hiller et al., Calibration of the absolute amplitude scale of the Tunka Radio Extension (Tunka-Rex), These proceedings (2015).

[4] LOFAR Collaboration, A.Nelles et al., Calibrating the absolute amplitude scale for air showers measured at LOFAR, Submitted to JINST (2015).

[5] E. Polisensky, LFmap: A Low Frequency Sky Map Generating Program., Long Wavelength Array (LWA) Memo Series 111 (2007).

[6] T. Huege, M. Ludwig, and C. W. James, Simulating radio emission from air showers with CoREAS, in American Institute of Physics Conference Series (R. Lahmann, T. Eberl, K. Graf, C. James, T. Huege, T. Karg, and R. Nahnhauer, eds.), vol. 1535 of American Institute of Physics Conference Series, pp. 128-132, May, 2013. 